\title{
Neutrophil-to-Lymphocyte Ratio in Colorectal Liver Metastases: Simply Prognostic or Clinically Relevant?
}

\author{
Eliza W. Beal, MD, and Jordan M. Cloyd, MD \\ The Ohio State University Wexner Medical Center and James Cancer Hospital, Columbus, OH
}

In recent years, there has been considerable interest in using inflammatory biomarkers to improve prognostication for patients with cancer. Typically available on standard serum laboratory tests, these investigations have led to an alphabet soup of potential markers: neutrophil-to-lymphocyte ratio (NLR), lymphocyte-to-monocyte ratio (LMR), systemic inflammation response index (SIRI), systemic immune inflammation index (SII), prognostic nutritional index (PNI), modified systemic inflammation score (mSIS), fibrinogen and neutrophil-to-lymphocyte ratio score (FNLR), albumin-to-fibrinogen ratio (AFR), platelet-lymphocyte ratio (PLR), fibrinogen-to-pre-albumin ratio (FPR), and more. Of these, NLR has become one of the most commonly studied due to its simplicity and cost-effectiveness that results from the inclusion of a complete blood count with differentiation in most patients' cancer workup. Previous studies have linked elevated NLR to worse long-term survival outcomes among patients with metastatic colorectal cancer (mCRC), but its clinical value in the management of patients with resectable colorectal liver metastases (CRLM) remains unproven.

In that context, Verter et al. performed a retrospective single-institutional analysis of all patients who underwent resection of CRLM between 2005 and 2017 to evaluate the association between preoperative NLR and overall (OS), disease-specific (DSS), and recurrence-free survival (RFS). ${ }^{1}$ After stratifying patients based on normal (NLR $\leq$ $3, n=231)$ or high (NLR $>3, n=53)$ NLR, they found that

(C) Society of Surgical Oncology 2021

First Received: 24 March 2021

Accepted: 9 April 2021;

Published Online: 21 April 2021

E. W. Beal, MD

e-mail: Eliza.Beal@osumc.edu the groups were relatively well matched on most clinicopathologic characteristics except for a higher rate of simultaneous primary resections in the high-NLR group despite similar rates of synchronous disease. On univariate analysis, elevated NLR was associated with decreased OS (HR 1.6, 95\% CI 1.1-2.3, $p=0.01$ ), decreased DSS (HR $1.6,95 \%$ CI $1.1-2.4, p=0.02$ ), and decreased RFS (HR $1.6,95 \%$ CI $1.0-1.9, p=0.049)$, and the associations with OS (HR 1.6, 95\% CI 1.1-2.3, $p=0.02$ ) and DSS (HR 1.6, $95 \%$ CI $1.0-1.9, p=0.03$ ) were maintained on multivariable analysis as well. Furthermore, using a competing risk analysis, elevated NLR was associated with increased risk of extrahepatic recurrence but not intrahepatic recurrence.

Limitations to the study by Verter et al. should be acknowledged. First, the study is retrospective, from a single institution, and has a relatively small sample size. Second, some pertinent clinical factors known to influence survival were not included, namely embryonic origin (i.e., right- versus left-sided cancer) and tumor mutation status. Third, the influence of neoadjuvant therapy (administered to $76 \%$ of patients in the study) on NLR values is unknown. The exact timing of the preoperative blood draw could have varied from 30 days prior (perhaps even during final cycle of chemotherapy) to the day of surgery, with unknown but potentially significant impact on the NLR value. Finally, these findings are not novel; the role of the NLR as a prognostic factor in patients undergoing resection for CRLM has been examined previously; For example, Halazun et al. demonstrated that NLR $>5$ was independently associated with decreased OS following resection of CRLM, ${ }^{2}$ while Giakoustidis et al. reported an increased risk of extrahepatic recurrence and decreased OS among patients with CRLM undergoing neoadjuvant therapy and resection $^{3}$ and McCluney et al. found that NLR predicted postoperative complications. ${ }^{4}$ 
More importantly, the clinical relevance of the findings in the study by Verter et al. remains unclear. While identifying prognostic factors is important for clarifying patient expectations and making informed treatment decisions, the OS durations were actually acceptable in both groups (NLR $\leq 3: 5.2$ years; NLR $>3: 3.8$ years). Indeed, while the authors propose using preoperative NLR "as a surrogate marker of aggressive systemic disease," the survival curves presented by Verter et al. did not diverge for several years. Given these observations, withholding potentially curative intent surgical resection of CRLMs clearly should not be advocated for patients with elevated NLR. In addition, while a commonly cited advantage of the NLR and other inflammatory biomarkers is their convenience and cost-effectiveness in that they are composed of elements that are typically measured routinely for all patients during their evaluation and management, it remains unclear how commonly physicians calculate and utilize these scores to inform patient prognosis in clinical practice.

Instead, future research should pivot from identifying simple prognostic associations to evaluating predictive biomarkers that individualize treatment decisions. Research in this arena is in its infancy. For example, in a study of patients with mCRC, both the NLR and PLR were shown to be associated with progression-free survival (PFS) in patients undergoing therapy with anti- epidermal growth factor receptor (EGFR) agents (cetuximab or panitumumab) or bevacizumab. ${ }^{5}$ A further study in mCRC patients demonstrated that the systemic immune inflammation index (SII), NLR, and PLR were associated with PFS and OS, and that patients with low NLR treated with chemotherapy and bevacizumab had improved PFS in comparison with those treated with chemotherapy alone. ${ }^{6}$ To our knowledge, studies using NLR to guide treatment decisions or as a component of treatment algorithms have not been performed but would be an important next step if this research is to be carried forward. The use of dynamic longitudinal measures of inflammation to evaluate response to therapy and guide future therapeutic decision-making should also be pursued.
In summary, the study by Verter et al. confirms the results of prior studies establishing the prognostic significance of preoperative NLR, similar to other inflammatory markers, in predicting the outcomes of patients with CRLM. However, given the retrospective study design and the excellent survival outcomes experienced by all patients, elevated preoperative NLR should not be considered a contraindication to surgical resection. Designing prospective studies that evaluate the clinical relevance of these biomarkers in individualizing treatment decision-making should be prioritized.

DISCLOSURES The authors declare no conflicts of interest.

\section{REFERENCES}

1. Verter E, Berger Y, Perl G, et al. Neutrophil-to-lymphocyte ratio predicts recurrence pattern in patients with resectable colorectal liver metastases. Ann Surg Oncol 2021. https://doi.org/10.1245/ s10434-021-10000-6.

2. Halazun KJ, Aldoori A, Malik HZ, Prasad KR, Toogood GJ, Lodge JPA. Elevated preoperative neutrophil to lymphocyte ratio predicts survival following hepatic resection for colorectal liver metastases. Eur J Surg Oncol. 2008;34(1):55-60.

3. Giakoustidis A, Neofytou K, Khan AZ, et al. Neutrophil to lymphocyte ratio predicts pattern of recurrence in patients undergoing liver resection for colorectal liver metastasis and thus the overall survival. J Surg Oncol. 2015;111:445-50.

4. McCluney SJ, Giakoustidis A, Segler A, Bissel J, Valente R, Hutchings RR, et al. Neutrophil: lymphocyte ratio as a method of predicting complications following hepatic resection for colorectal liver metastasis. J Surg Oncol. 2018;117(5):1058-65.

5. Dogan E, Bozkurt O, Sakalar T, Derin S, Inanc M, Ozkan M. Impact of neutrophil-lymphocyte and platelet-lymphocyte ratio on antiEGFR and bevacizumab efficacy in metastatic colorectal cancer. J BUON . 2019;24(5):1861-9.

6. Passardi A, Scarpi E, Cavanna L, Dall'Agata M, Tassinari D, Leo $S$, et al. Inflammatory indexes as predictors of prognosis and bevacizumab efficacy in patients with metastatic colorectal cancer. Oncotarget. 2016;7(22):33210-9.

Publisher's Note Springer Nature remains neutral with regard to jurisdictional claims in published maps and institutional affiliations. 